\title{
RdRp mutations are associated with SARS-CoV-2 genome evolution
}

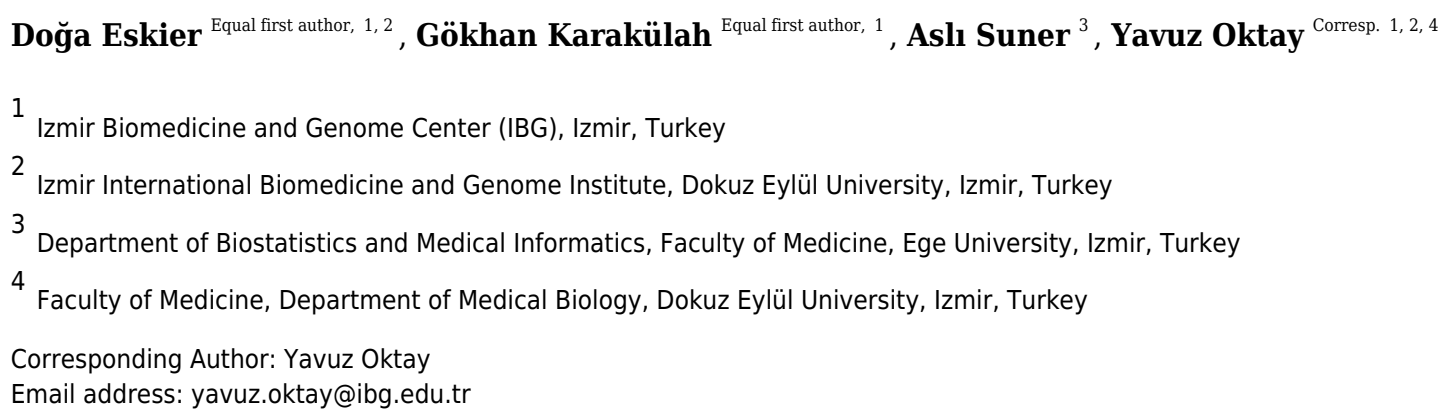

COVID-19, caused by the novel SARS-CoV-2 virus, started in China in late 2019, and soon became a global pandemic. With the help of thousands of viral genome sequences that have been accumulating, it has become possible to track the evolution of viral genome over time as it spread across the world. An important question that still needs to be answered is whether any of the common mutations affect the viral properties, and therefore the disease characteristics. Therefore, we sought to understand the effects of mutations in RNA-dependent RNA polymerase (RdRp), particularly the common $14408 \mathrm{C}>\mathrm{T}$ mutation, on mutation rate and viral spread. By focusing on mutations in the slowly evolving $\mathrm{M}$ or $\mathrm{E}$ genes, we aimed to minimize the effects of selective pressure. Our results indicate that $14408 \mathrm{C}>\mathrm{T}$ mutation increases the mutation rate, while the third-most common RdRp mutation, $15324 \mathrm{C}>\mathrm{T}$, has the opposite effect. It is possible that $14408 \mathrm{C}>\mathrm{T}$ mutation may have contributed to the dominance of its co-mutations in Europe and elsewhere. 


\section{RdRp mutations are associated with SARS-CoV-2 genome evolution}

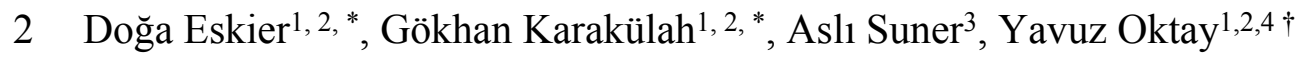

$4 \quad{ }^{1}$ Izmir Biomedicine and Genome Center, Izmir, Turkey

$5 \quad{ }^{2}$ Izmir International Biomedicine and Genome Institute, Dokuz Eylül University, Izmir, Turkey

$6 \quad{ }^{3}$ Department of Biostatistics and Medical Informatics, Faculty of Medicine, Ege University, Izmir,

7 Turkey

$8 \quad{ }^{4}$ Faculty of Medicine, Department of Medical Biology, Dokuz Eylül University, Izmir, Turkey

$9{ }^{*}$ These authors contributed equally to the work presented here and should therefore be regarded as

10 first authors.

11 Corresponding author: Yavuz Oktay, E-mail: yavuz.oktay@ibg.edu.tr

12 


\section{Abstract}

14 COVID-19, caused by the novel SARS-CoV-2 virus, started in China in late 2019, and soon

15 became a global pandemic. With the help of thousands of viral genome sequences that have been

16 accumulating, it has become possible to track the evolution of viral genome over time as it

17 spread across the world. An important question that still needs to be answered is whether any of

18 the common mutations affect the viral properties, and therefore the disease characteristics.

19 Therefore, we sought to understand the effects of mutations in RNA-dependent RNA polymerase

20 (RdRp), particularly the common 14408C $>$ T mutation, on mutation rate and viral spread. By

21 focusing on mutations in the slowly evolving $\mathrm{M}$ or $\mathrm{E}$ genes, we aimed to minimize the effects of

22 selective pressure. Our results indicate that $14408 \mathrm{C}>\mathrm{T}$ mutation increases the mutation rate,

23 while the third-most common RdRp mutation, $15324 \mathrm{C}>\mathrm{T}$, has the opposite effect. It is possible

24 that $14408 \mathrm{C}>\mathrm{T}$ mutation may have contributed to the dominance of its co-mutations in Europe

25 and elsewhere.

26 Keywords: SARS-CoV-2, COVID-19, RNA-dependent RNA polymerase, RdRp, mutation rate

\section{Introduction}

28 SARS-CoV-2 is a novel betacoronavirus originally identified in December 2019, and given the

29 official name on 11 February 2020. It is responsible for the ongoing COVID-19 pandemic, with

30 the earliest known patients located potentially as early as November 2019, in the Hubei province

31 of China. Human to human transmission of the virus was confirmed on 20 January 2020 (Chan et

32 al., 2020), with 6 deaths and 282 confirmed cases on 21 January 2020, which, as of 13 May

33 2020, had respectively increased to over 283 thousand deaths and 4.09 million cases, with a

34 projected mortality rate of $<7 \%$, and an R0 number estimation of $1.4-3.8$ (Riou \& Althaus, 
35 2020). Due to its high transmission rate, and worldwide distribution of known cases, SARS-

$36 \mathrm{CoV}-2$ is a high priority for medical research despite the low mortality rate. Furthermore, new

37 COVID-19 symptoms continue to be discovered in even recovering patients (Wilson,

38 Katlariwala \& Low, 2020; Li et al., 2020), making it difficult to fully understand the global

39 impact of the disease. To date, there is no known targeted treatment or vaccine for SARS-CoV-2.

40 The current reference genome for SARS-CoV-2, NC_045512.2, was sequenced in Shanghai,

41 China, and submitted on 5 January 2020, replacing the previous reference sequence on 17

42 January 2020. SARS-CoV-2 has a 29903-nucleotide long single stranded sense RNA genome,

43 which codes for 12 peptides, including two closely related polyproteins, Orfla and Orf1ab,

44 which are further cleaved into 26 mature peptides, and the main structural proteins, such as the

45 surface glycoprotein, nucleocapsid phosphoprotein, membrane glycoprotein, and envelope

46 glycoprotein (genes S, N, M, and E, respectively)(“Severe acute respiratory syndrome

47 coronavirus 2 isolate Wuhan-Hu-1, complete genome," 2020). The primary binding target of

48 SARS-CoV-2 surface glycoprotein for entry into the human cell is the ACE2 protein (Tian et al.,

49 2020), localized in the cell membrane in a number of tissues (Hikmet et al., 2020). It is predicted

50 that the virus is zoonotic in origin, and a mutation in the surface glycoprotein structure enabled

51 transmission to human hosts. As a result of the origins of the disease, and due to the targeted

52 nature of vaccine and drug discovery efforts, identifying the replication-related mutation rate and

53 the global mutatome of SARS-CoV-2 is crucial to efforts in combating the disease. Despite

54 having proof-reading capability, analyses of SARS-CoV-2 genomes indicated nucleotide

55 substitution rates comparable to other RNA viruses that lack such capability (Zhang \& Holmes,

56 2020). It is difficult to pinpoint the underlying causes without functional studies; however, one 
57 plausible explanation would be reduced fidelity of the main RNA polymerase, namely RNA-

58 dependent RNA polymerase ( $R d R p$, also known as nsp12), due to mutations.

59 SARS-CoV-2 does not depend on host polymerases to replicate its genome, instead using the

$60 \mathrm{RdRp}$ and associated proteins (i.e. nsp7, nsp8, and nsp14, the latter an exonuclease with error

61 correction capabilities), all of which are encoded by its own genome (Subissi et al., 2014; Ma et

62 al., 2015). Among the SARS-CoV-2 isolates from all over the world, several widespread

63 mutations on the RdRp coding region of the Orflab polyprotein gene have been identified. Key

64 among them is the $14408 \mathrm{C}>\mathrm{T}$ transition, identified in over 7000 isolates across multiple

65 continents. In an early analysis of 137 SARS-CoV-2 genomes from North America and Europe,

66 Pachetti et al. suggested that this particular mutation is associated with higher number of

67 mutations, through a mechanism not yet fully known (Pachetti et al., 2020). The proline to

68 leucine substitution (P323L) caused by the $14408 \mathrm{C}>\mathrm{T}$ mutation has been suggested to rigidify

69 the RdRp protein structure, which may exert its effects through altered interaction with other

70 components of the replication / transcription machinery or with the RNA template, and thereby

71 resulting in an altered mutation rate (Pachetti et al., 2020; Begum et al., 2020). However, further

72 studies are needed to test these hypotheses.

73 Although the higher number of mutations in genomes with RdRp 14408C $>$ T mutation was

74 suggested to be caused by lower fidelity of the mutant enzyme, it could also be due to many

75 other epidemiological factors, such as worse containment of the mutant strain compared to

76 others, more frequent introduction of the mutant strain into high-risk and/or higher-transmitting

77 populations by chance, etc. Importantly, natural selection acting on different levels and ways in

78 different environments could potentially lead to faulty estimates of any change in the 
79 replicational mutation rate. Therefore, in order to minimize the effects of natural selection, we

80 focused our attention on parts of the genome that may be under lower selective pressure.

81 A recent study by Dilucca et al. showed that different SARS-CoV-2 genes are under varying

82 levels of selective pressure, with $\mathrm{M}$ and $\mathrm{E}$ integral proteins being subject to relatively low natural

83 selection and a low, non-selective mutation rate, largely as a result of accumulation of replication

84 errors. On the other hand, key proteins for virulence and transmissibility, such as the S protein,

85 seem to be under high selective pressure, possibly as a result of novel host adaptation (Dilucca et

86 al., 2020). To identify how the RdRp mutations affect the mutation rate of the SARS-CoV-2

87 genome, we examined the relationships of the RdRp mutations with mutations found in $\mathrm{M}$ or $\mathrm{E}$

88 proteins (hereafter referred to as MoE), in terms of both time and location. In particular, we

89 focused on the 10 most common mutations in the RdRp region, with the goal of identifying

90 whether each variant is associated with increased or decreased non-selective mutation rates, and

91 whether the geographical distribution of these variants might suggest the presence of multiple

92 forms of virus with various mutability across the globe.

\section{Materials and Methods}

94 Genome sequence filtering, retrieval, and preprocessing

95 SARS-CoV-2 isolate genome sequences were obtained from the GISAID EpiCoV database

96 ("GISAID Initiative"). The genomes were filtered for those obtained from human hosts, a

97 sequence length of at least 29000 nucleotides, and high coverage $(<1 \%$ undefined nucleotides, $<$

$980.05 \%$ mutations not seen in any other isolate, and no indel mutations that were not verified by

99 the submitter). The filters resulted in a total of 11901 remaining genomes (as of May 5th, 2020).

100 The genomes were aligned against the reference genome sequence obtained from the NCBI

101 Nucleotide database in the FASTA format, under locus ID NC_045512.2 
102 (https://www.ncbi.nlm.nih.gov/nuccore/NC 045512.2), after nonstandard unresolved base calls

103 (sequence characters which are not A, C, G, T, N, or -) were changed into the standard

104 unresolved sequence character $\mathrm{N}$ via the Unix sed command. The alignment was performed with

105 the MAFFT multiple sequence alignment program, using the "--auto --keeplength --

106 addfragments isolate_genomes.fa reference_genome.fa $>$ alignment.fa" parameters. The sites

107 differing from the reference sequence were extracted using snp-sites (https://github.com/sanger-

108 pathogens/snp-sites), with the "-v -o variants.vcf alignment.fa" options. The resulting VCF file

109 was modified for compatibility with the following steps using text editing and bcftools

110 (http://www.htslib.org/download/), replacing the first column, indicating reference sequence

111 name, with NC_045512v2, and separating different variants at the same nucleotide to individual

112 lines, using the VCF processing guide available in the ANNOVAR documentation (https://doc-

113 openbio.readthedocs.io/projects/annovar/en/latest/articles/VCF/). The final VCF file was

114 converted into an avinput file, using convert2annovar.pl found under ANNOVAR, with the

115 parameters "-format vcf4old variants.vcf > variants.avinput". The custom ANNOVAR gene

116 annotations for SARS-CoV-2 were obtained from ANNOVAR resources, decompressed, and

117 placed in the sarscov2db directory. The variants were then annotated in terms of their

118 relationships to gene loci and products, using the table_annovar.pl function of ANNOVAR, with

119 the parameters “-buildver NC_045512v2 variants.avinput sarscov2db/ -protocol avGene -

120 operation g”.

121 Following the alignment and annotation, the 5' untranslated region of the genome (bases 1-265)

122 and the 100 nucleotides at the 3 ' end were removed from analysis due to lack of quality

123 sequencing in a majority of isolates. To ensure a vigorous examination of the association of both

124 time and location and the mutations, we have further filtered out isolate genomes without well- 
125 defined time of sequencing metadata (year - month - day), and an undefined geographical

126 location, for a final count of 11208 genomes. 71 of these genomes were sequenced in Africa, 859

127 were sequenced in Asia, 5769 in Europe, 3370 in North America, 1021 in Oceania, and 118 in

128 South America.

129 Statistical Analysis

130 Descriptive statistics for continuous variable days were calculated with mean, standard deviation,

131 median, and interquartile range. Shapiro-Wilk test was used to check the normality assumption

132 of the continuous variable. In cases of non-normally distributed data, the Wilcoxon rank-sum

133 (Mann-Whitney U) test was performed to determine whether the difference between the two

134 MoE groups was statistically significant. The Fisher's exact test and the Pearson chi-square test

135 were used for the analysis of categorical variables. The univariate logistic regression method was

136 utilized to assess the mutations associated with MoE in single variables, and then multiple

137 logistic regression method was performed. The final multiple logistic regression model was

138 executed with the backward stepwise method. A p-value of less than 0.05 was considered

139 statistically significant. All statistical analyses were performed using IBM SPSS version 25.0

140 (Chicago, IL, USA).

141

142 Results and Discussion

143 Mutation profile of SARS-CoV-2 genome as of 5 May 2020

144 After the low quality filters were applied, 5658 nucleotides, making up $18.9 \%$ of the SARS-

145 CoV-2 genome, were found to carry a mutation in at least one isolate, with 2668 of these sites

146 being mutated in multiple isolates. The sites mutated in at least two isolates had a mean of 26.25

147 mutated isolates, and a median of 3. To identify the distribution of common mutations by the 
148 number of isolates they are found in, we identified the top 50 mutated sites and the number of

149 isolates with a non-reference resolved base in those sites (Fig. 1). Three nucleotides, 3037,

15014408 , and 23403, were found to be mutated in over 7000 isolates. Out of these three, $14408 \mathrm{C}>\mathrm{T}$

151 was previously established as a mutation of interest for the RdRp gene. $23403 \mathrm{~A}>\mathrm{G}$ is a

152 nonsynonymous mutation in the surface glycoprotein, while 3037 is a synonymous mutation in

153 nsp3, a replication scaffolding protein (Yin, 2020). Notably, only 26 of the top 50 sites were

154 found in the Orflab coding region, despite it comprising 71\% of the SARS-CoV-2 genome, with

155 the percentage being $57 \%, 61 \%$, and $65.3 \%$ when we consider top 100 , top 200 , and all mutated

156 sites, respectively. Other than $14408 \mathrm{C}>\mathrm{T}$, two of the top 50 mutations were also in the RdRP

157 coding sequence, although both of them are synonymous mutations that do not presumably affect

158 the protein structure. Two of the top 50 sites were found in the membrane glycoprotein coding

159 region, with $26729 \mathrm{~T}>\mathrm{C}$ being a synonymous mutation, and $27046 \mathrm{C}>\mathrm{T}$ being a nonsynonymous

160 mutation causing a T175M mutation in the peptide sequence. None of the top 50 mutated sites

161 were found in the envelope glycoprotein region, which has only 23 sites mutated in multiple

162 isolates.

163 We then examined the distribution of mutant RdRp, envelope, and membrane protein genes by

164 geographical location (Africa, Asia, Europe, North America, Oceania, and South America), in

165 order to see what percentage of isolates from each region carried a mutation in these coding

166 regions. South America had the highest percentage of RdRp mutants, 93.22\%, while Asia had

167 only $32.71 \%$, the lowest among the regions. South America also had the highest number of

168 mutant isolates for the $\mathrm{M}$ gene, and the second highest for the $\mathrm{E}$ gene, at $11.02 \%$ and $2.54 \%$

169 respectively (Fig. 2). However, it should be noted that Africa and South America both have

170 remarkably lower number of sequences available and the disease showed a relatively late spread 
171 in these two continents compared to others. The first case in South America (EPI_ISL_412964)

172 was a 61 year-old male who travelled to Northern Italy and returned on February 21. This

173 patient-zero carried the SARS-CoV-2 strain dominant in Italy and led to local transmission,

174 which may explain the high-frequency of mutations at nucleotides 3037, 14408 and 23403 in

175 Brazil. Interestingly, a few other cases who also returned from Northern Italy within a few days

176 of patient-zero did not spread the disease as effectively. On the other hand, high-frequency of

177 isolates with $\mathrm{M}$ or E mutations in South America can possibly be explained by relatively late

178 spread of the disease (“GISAID Initiative").

\section{Associations between MoE and top ten frequently observed mutations in RdRp}

180 To examine how the most common mutations in RdRp affect mutation rate of the SARS-CoV-2

181 genome, we identified the 10 most frequently mutated nucleotides in the RdRp region. Table 1

182 summarizes the frequencies and comparisons of MoE between RdRp mutants. There are

183 statistically significant associations between the mutations at nucleotides $13730,14408,14805$,

18415324 and $\mathrm{MoE}(\mathrm{p}<0.05)$. However, our statistical analysis indicates that there are no significant

185 associations between the mutations 13536, 13627, 13862, 14786, 14877, 15540 and MoE

$186(\mathrm{p}>0.05)$.

187 We also tested for possible associations between MoE and time (in days). Mean days in the MoE

188 absent and present groups were $85.80 \pm 15.56$, and $85.77 \pm 15.61$, respectively. The days variable

189 in the MoE absent group was not statistically significantly higher than the MoE present group

190 (Median (IQR): 88.00 (15) versus $86.00(18) ; \mathrm{p}=0.095)$. Because it was not statistically

191 significant, we did not include 'days' in the logistic regression models. 


\section{Associations between MoE and geographic locations}

193 Distribution of SARS-CoV-2 mutations show variability among geographical locations, mainly

194 due to founder effects, as well as various other epidemiological factors. In order to compare the

195 distribution of MoE among different geographic locations, Table 2 shows that there are

196 statistically significant associations between the locations and $\operatorname{MoE}(\mathrm{p}<0.001)$. The most

197 frequently observed location for the MoE is Europe $(n=658)$, however, it is largely due to higher

198 representation of European viral genomes in the GISAID database. The highest proportion of

199 MoE is seen in South America (12.7), whereas North America has the lowest (5.2\%).

\section{Logistic regression models of the MoE}

201 Next, we evaluated location in the univariate logistic regression models of the MoE (absent (0)

202 and present (1)) for each location (itself (1) and others (0)) (Table 3). Europe, North America and

203 Oceania were found statistically significant to predict $\operatorname{MoE}(\mathrm{p}<0.05)$. In these locations, while

204 the odds ratio for Europe was 1.700 (95\% CI, 1.490-1.939; p<0.001), odds ratios for North

205 America and the Oceania were 0.444 (95\% CI, 0.376-0.525; $\mathrm{p}<0.001)$ and $1.297(95 \%$ CI,

206 1.058-1.591; $\mathrm{p}=0.012$ ) for the presence of MoE. Thus, our results suggest that SARS-CoV-2

207 genomes in Europe and Oceania are more likely to have MoE compared to other locations (1.7

208 and 1.3 times, respectively), while those in North America are $>2.2$ times less likely.

209 In the univariate logistic regression models of the MoE (absent (0) and present (1)), when the ten

210 mutations were included separately in the models, 14408, 14805, 15324, and 13730 were found

211 statistically significant to predict $\operatorname{MoE}(\mathrm{p}<0.05)$ (Table 4). In the final model (Final Model A),

212 significant associations were also detected between MoE and these four mutations $(\mathrm{p}<0.05)$. In 
213 the final model of the four mutations, the odds ratio for 14408 was $1.522(95 \%$ CI, $1.305-1.776$;

$214 \mathrm{p}<0.001)$ for the MoE. Thus, our results suggest that SARS-CoV-2 genomes with the $14408 \mathrm{C}>\mathrm{T}$

215 mutation are 1.5 times more likely to have MoE. We also evaluated 'location' in the univariate

216 logistic regression models of the $\mathrm{MoE}$, and found that it was statistically significant to predict

$217 \operatorname{MoE}(\mathrm{p}>0.001)$. Therefore, the final model of logistic regression analysis for independent

218 variables 14408, 14805, 15324, 13730 and 'location' (Asia is the reference group) was then built

219 to evaluate their associations with MoE (Final Model B). This final analysis revealed that the

220 same four mutations $(14408,14805,15324,13730)$ and 'location' were significantly associated

221 with $\mathrm{MoE}(\mathrm{p}<0.05)$. For the location, Europe, North America and Oceania were found to be

222 statistically significant on single variable model for the location to predict $\mathrm{MoE}(\mathrm{p}=0.003$,

$223 \mathrm{p}=0.002$, and $\mathrm{p}=0.014$, respectively), similarly same locations were also statistically significant

224 in the final model $(p=0.036, p<0.001$, and $p=0.026$, respectively). According to the final model

225 that was constructed with four mutations and location (Final Model B), viral genomes both in

226 Europe and Oceania are more likely to have MoE compared to genomes in Asia (1.35 and 1.45

227 times, respectively). These results indicate that both RdRp mutations and location independently

228 predict MoE status.

229 Whereas the $14408 \mathrm{C}>\mathrm{T}$ mutation predicted higher risk of MoE, the other three significant

230 mutations in $\mathrm{RdRp}$ predicted a lower risk, particularly the 15324C $>$ T mutation, which predicted

231 about 10-fold reduced risk of MoE. Although location was another predictor of MoE, as

232 expected, multivariate logistic regression analysis indicated that the association between RdRp

233 mutations and mutational status of $\mathrm{M}$ or E genes was independent from location. 
234 Two of the four significant RdRp mutations were first detected around the same time, 15324 on

235 January 22, and 14408 on January 24, both in Asia. 14805 was first detected in a European

236 genome on February 9, and the most recent of the four, 3730 was detected in an Asian genome

237 on March 4. Despite arising within a few days interval (based on first genomes which have been

238 detected in so far), 14408 and 15324 display $>20$-fold difference in their spread: $14408(\mathrm{n}=7015$

239 genomes) vs. 15324 ( $\mathrm{n}=305$ genomes). Although this observation may be explained by better

240 adaptation of the viruses with a mutation that cause increased mutation rate to changing

241 environments, it could as well be explained by founder effects, genetic drift, and other

242 epidemiological factors. More data and particularly functional studies where mutant viruses can

243 be compared side by side will be required to test this hypothesis.

244 Our observation that the two different mutants of RdRp result in $\sim 14$-fold difference in the

245 likelihood of having a mutation in parts of the genome that evolves relatively slow and under less

246 selective pressure ( $\mathrm{M}$ and E genes) supports the hypothesis that mutations of RdRp contribute

247 significantly to the SARS-CoV-2 genome evolution. A mutant RdRp that is more error-prone

248 would be expected to increase viral genetic diversity and allow the virus spread under different

249 selective pressures, such as spreading to different populations. As lower-fidelity is also

250 associated with higher speed, such mutations may also allow higher titers of virus within host

251 cells. On the other hand, a higher-fidelity polymerase would be suitable where optimal

252 conditions are reached and errors in replication would be costly. Although preliminary studies

253 suggest that $14408 \mathrm{C}>\mathrm{T}(\mathrm{P} 323 \mathrm{~L}$ in $\mathrm{RdRp})$ could lower replication fidelity, it is less clear how the

254 synonymous $15324 \mathrm{C}>\mathrm{T}$ mutation could lead to lower mutation rates ${ }^{12}$. It should be noted that

255288 of $305(94.4 \%)$ genomes worldwide with the $15324 \mathrm{C}>\mathrm{T}$ mutation also have the $14408 \mathrm{C}>\mathrm{T}$

256 mutation, and MoE rate is $1.39 \%$ (4/288) among double mutants, whereas it is $11.13 \%$ 
257 (749/6727) for $14408 \mathrm{C}>\mathrm{T}$-only mutants. It is possible that $15324 \mathrm{C}$ is part of an as-yet-unknown

258 viral sequence that interacts with host factor(s) and $15324 \mathrm{C}>\mathrm{T}$ mutation indirectly affects the

$25914408 \mathrm{C}>\mathrm{T}$ mutation through modulation of this interaction; as there are currently only 305

260 genome sequences available with this mutation, this question may be better answered as more

261 viral genome sequences accumulate and functional studies are performed.

262 Three other mutations that co-evolved and seen together with $14408 \mathrm{C}>\mathrm{T}$ are $23403 \mathrm{~A}>\mathrm{G}$ (D614G

263 in S protein) and 3037C $>$ T (F106F in NSP3). The first 14408C $>$ T mutation dates to a patient

264 whose sample was collected on January 24 in China, but sequenced and submitted to GISAID on

265 April 10. However, it took 27 days for the second case with the same mutation to appear, and

266 interestingly, not in China, but in Italy. Two days later, on February 22, the first case with

$26714408 \mathrm{C}>\mathrm{T}$ was reported in Australia, 31 days after the first SARS-CoV-2 case in the country.

268 Following its introduction to Europe, it took another 10 days for the emergence of the second

269 case in Asia, which can be possibly attributed to strict measures taken by the authorities that led

270 to a steep decline in viral spread particularly in China. In contrast to Europe, North and South

271 America, where $14408 \mathrm{C}>\mathrm{T}$ became the dominant form together with its co-mutations

$272(23403 \mathrm{~A}>\mathrm{G}$ and $3037 \mathrm{C}>\mathrm{T}), 14408 \mathrm{C}>\mathrm{T}$ and its co-mutations remained as the minor form in Asia,

$27314408 \mathrm{C}>\mathrm{T}$ being present in only 15.9\% (137/859) of viral genomes. Emergence of $14408 \mathrm{C}>\mathrm{T}$ in

274 South America, North America and Africa was 5, 7 and 8 days following the first European

275 mutant virus, and again became the dominant form, as $81.3 \%, 59.4 \%$ and $80.3 \%$ of viral

276 genomes carry the mutation, respectively.

277 A recent study postulated that the one of the co-mutations of $14408 \mathrm{C}>\mathrm{T}$, namely $23403 \mathrm{~A}>\mathrm{G}$ that

278 causes D614G mutation in the S protein may result in a more transmissible form of SARS-CoV- 
2792 (Korber et al., 2020). This claim was based mainly on the observation that D614G mutant virus

280 became the dominant form in more than one geographical location upon its introduction, as

281 summarized above for its co-mutation $14408 \mathrm{C}>\mathrm{T}$. However, in the absence of mechanistic

282 insight to explain how this particular mutation could lead to higher transmissibility, other

283 explanations based on stochastic factors are equally possible. A study by Bhattacharyya et al.

284 suggested that D614G mutation creates an additional protease cleavage site near the S1-S2

285 junction, which may increase the success of viral integration with the host cell, and linked its

286 dominance in Europe to certain human variants that control expression of TMPRSS2

287 (Bhattacharyya et al., 2020).

288 On the other hand, it is intriguing that between the first appearance of three co-mutations (on

$28914408,23403,3037)$ on January 24 in a Chinese isolate (EPI_ISL_422425) and their second co-

290 appearance on February 20 in an Italian isolate (EPI_ISL_412973), there are at least 6 and

291 possibly 8 different viral genomes where three of the four co-mutations exist, with the exception

292 of 14408C>T: on January 28 in Germany (EPI_ISL_406862), on February 5, 6, 7 and 8 in China

293 (EPI_ISL_429080, EPI_ISL_429081, EPI_ISL_416334, EPI_ISL_412982, and

294 EPI_ISL_429089); and two more Chinese viral sequences that failed our quality control

295 standards and therefore eliminated from the overall analysis. Despite weeks of existence,

$29623403 \mathrm{~A}>\mathrm{G}$ became the dominant form only after the appearance of the first Italian case with all

297 four mutations on February 20. If this form of SARS-CoV-2 is really more transmissible, the

298 next question that needs to be answered is whether it is due to any one of the three mutations

299 alone, or whether a combination of two or three are needed. Based on the lack of successful

300 spreading of the virus in its absence and our results showing increased mutability in its presence,

301 we speculate that $14408 \mathrm{C}>\mathrm{T}$ could be cooperating with the other two mutations. Alternatively, 
302 altered mutation rate may be a byproduct and the RdRp mutations may act through speeding up

303 or slowing down the replication process, which would in turn affect the viral load and virulence.

304 Also, viruses with mutant RdRp may become more resistant to anti-viral drugs, such as the

305 commonly used remdesivir. Such implications make RdRp mutations attractive targets for

306 epidemiological and functional studies with direct therapeutic implications.

307 Conclusions

308 Effects of different mutations on SARS-CoV-2 phenotypes (i.e. mutation rate, transmissibility,

309 virulence, immune evasion etc.) are hot topics of research as there is an intense race worldwide

310 to develop therapies and understand the viral biology. Some of these studies suggested that RdRp

311 and spike protein mutations could significantly affect the virus behavior and therefore the human

312 health. Our study sheds light on the effects RdRp mutations, particularly $14408 \mathrm{C}>\mathrm{T}$ mutation, on

313 the mutability and possibly transmissibility of SARS-CoV-2. Further functional studies are

314 required to test our findings.

\section{Additional Information and Declarations}

\section{Acknowledgement}

317 The authors would like to thank Mr. Alirıza Arıbaş and Mr. Ahmet Bursalı from Izmir Biomedicine

318 and Genome Center for their technical assistance. The authors also would like to extend their

319 thanks to Izmir Biomedicine and Genome Center (IBG) COVID19 platform IBG-COVID19 for

320 their support in implementing the study and the Scientific and Technological Research Council of

321 Turkey (TUBITAK) for their financial support of IBG-COVID19.

\section{Funding}


323 YO is supported by the Turkish Academy of Sciences Young Investigator Program (TÜBA-

\section{GEBİP)}

\section{Grant Disclosures}

326 Not applicable

\section{Competing Interests}

328 AS and GK are associate editors in PeerJ.

\section{Author Contributions}

330 - DE conceived and designed the experiments, performed the experiments, analyzed the data,

331 contributed reagents/materials/analysis tools, prepared figures and/or tables, authored or

332 reviewed drafts of the paper, approved the final draft.

333 - GK conceived and designed the experiments, performed the experiments, analyzed the data,

334 contributed reagents/materials/analysis tools, prepared figures and/or tables, authored or

335 reviewed drafts of the paper, approved the final draft.

336 - AS conceived and designed the experiments, performed the experiments, analyzed the data,

337 contributed reagents/materials/analysis tools, prepared figures and/or tables, authored or

338 reviewed drafts of the paper, approved the final draft.

339 -YO conceived and designed the experiments, performed the experiments, analyzed the data,

340 contributed reagents/materials/analysis tools, prepared figures and/or tables, authored or

341 reviewed drafts of the paper, approved the final draft. 


\section{Data Availability}

343 The processed genotyping VCF file is available here: doi:10.17632/x4t94w9njt.1

\section{Supplemental Information}

345 Not applicable

\section{References}

347 Begum F, Mukherjee D, Das S, Thagriki D, Tripathi PP, Banerjee AK, Ray U. 2020. Specific

348 mutations in SARS-CoV2 RNA dependent RNA polymerase and helicase alter protein

349 structure, dynamics and thus function: Effect on viral RNA replication. Molecular

$350 \quad$ Biology. DOI: 10.1101/2020.04.26.063024.

351 Bhattacharyya C, Das C, Ghosh A, Singh AK, Mukherjee S, Majumder PP, Basu A, Biswas NK. 2020. Global Spread of SARS-CoV-2 Subtype with Spike Protein Mutation D614G is Shaped by Human Genomic Variations that Regulate Expression of TMPRSS2 and MX1 Genes. Genomics. DOI: 10.1101/2020.05.04.075911.

Chan JF-W, Yuan S, Kok K-H, To KK-W, Chu H, Yang J, Xing F, Liu J, Yip CC-Y, Poon RWS, Tsoi H-W, Lo SK-F, Chan K-H, Poon VK-M, Chan W-M, Ip JD, Cai J-P, Cheng VC-

Dilucca M, Forcelloni S, Georgakilas AG, Giansanti A, Pavlopoulou A. 2020. Codon Usage and 10.3390/v12050498. 
363 GISAID Initiative. Available at https://www.epicov.org/epi3/frontend\#272e13 (accessed May 19, 364 2020).

365 Hikmet F, Méar L, Edvinsson Å, Micke P, Uhlén M, Lindskog C. 2020. The protein expression 366 profile of ACE2 in human tissues. bioRxiv:2020.03.31.016048. DOI: 10.1101/2020.03.31.016048.

368

369

370

371

372

373

374

375

376

377

378

379

380

381

382

383

384

385

Korber B, Fischer WM, Gnanakaran S, Yoon H, Theiler J, Abfalterer W, Foley B, Giorgi EE, Bhattacharya T, Parker MD, Partridge DG, Evans CM, Freeman TM, Silva T de, Group on behalf of the SC-19 G, LaBranche CC, Montefiori DC. 2020. Spike mutation pipeline reveals the emergence of a more transmissible form of SARS-CoV-2. bioRxiv:2020.04.29.069054. DOI: 10.1101/2020.04.29.069054.

Li J, Long X, Zhang Q, Fang X, Fang F, Lv X, Zhang D, Sun Y, Li N, Hu S, Lin Z, Xiong N. 2020. Emerging evidence for neuropsycho-consequences of COVID-19. Current Neuropharmacology. DOI: 10.2174/1570159X18666200507085335.

Ma Y, Wu L, Shaw N, Gao Y, Wang J, Sun Y, Lou Z, Yan L, Zhang R, Rao Z. 2015. Structural basis and functional analysis of the SARS coronavirus nsp14-nsp10 complex. Proceedings of the National Academy of Sciences of the United States of America 112:9436-9441. DOI: 10.1073/pnas.1508686112.

Pachetti M, Marini B, Benedetti F, Giudici F, Mauro E, Storici P, Masciovecchio C, Angeletti S, Ciccozzi M, Gallo RC, Zella D, Ippodrino R. 2020. Emerging SARS-CoV-2 mutation hot spots include a novel RNA-dependent-RNA polymerase variant. Journal of Translational Medicine 18:179. DOI: 10.1186/s12967-020-02344-6.

Riou J, Althaus CL. 2020. Pattern of early human-to-human transmission of Wuhan 2019 novel coronavirus (2019-nCoV), December 2019 to January 2020. Euro Surveillance: Bulletin 

25. DOI: $10.2807 / 1560-7917 . E S .2020 .25 .4 .2000058$.

388 Severe acute respiratory syndrome coronavirus 2 isolate Wuhan-Hu-1, complete genome. 2020. Available at http://www.ncbi.nlm.nih.gov/nuccore/NC_045512.2 (accessed May 15, 2020).

391
Subissi L, Posthuma CC, Collet A, Zevenhoven-Dobbe JC, Gorbalenya AE, Decroly E, Snijder EJ, Canard B, Imbert I. 2014. One severe acute respiratory syndrome coronavirus protein complex integrates processive RNA polymerase and exonuclease activities. Proceedings of the National Academy of Sciences 111:E3900-E3909. DOI: 10.1073/pnas.1323705111.

Tian X, Li C, Huang A, Xia S, Lu S, Shi Z, Lu L, Jiang S, Yang Z, Wu Y, Ying T. 2020. Potent binding of 2019 novel coronavirus spike protein by a SARS coronavirus-specific human monoclonal antibody. Emerging Microbes \& Infections 9:382-385. DOI: 10.1080/22221751.2020.1729069.

Wilson MP, Katlariwala P, Low G. 2020. Potential implications of novel coronavirus disease (COVID-19) related gastrointestinal symptoms for abdominal imaging. Radiography (London, England: 1995). DOI: 10.1016/j.radi.2020.04.016.

Yin C. 2020. Genotyping coronavirus SARS-CoV-2: methods and implications. Genomics. DOI: 10.1016/j.ygeno.2020.04.016.

Zhang Y-Z, Holmes EC. 2020. A Genomic Perspective on the Origin and Emergence of SARSCoV-2. Cell 181:223-227. DOI: 10.1016/j.cell.2020.03.035. 


\section{Figure Legends}

\section{Figure 1.}

411 Bar graph of top 50 most mutated nucleotides vs. log2-transformed number of samples with non-

412 reference nucleotide at position. The $\mathrm{x}$-axis represents the position of the nucleotide in the

413 reference genome, the y-axis represents $\log 2$ of number of isolates with disagreeing nucleotide

414 aligning to the position in sequence plus 1 . Unresolved sequence calls during library sequencing

415 or gaps are not included in the number of isolates. The 5' untranslated region and the 3'-most

416100 nucleotides are not included in the graph.

\section{Figure 2.}

418 Bar graph of percent of isolates per region containing non-reference coding sequences for

419 envelope glycoprotein (E), membrane glycoprotein (M), and RdRp protein. Violet bars represent

420 percent of isolates with mutant envelope glycoprotein, while orange bars represent the same

421 percentage for membrane glycoprotein, and green bars represent the same for RdRp. 


\section{Table 1 (on next page)}

Comparisons of MoE and RdRp mutations.

*p-value $<0.05$ was statistically significant. 
Table 1. Comparisons of $\mathrm{MoE}$ and RdRp mutations.

\begin{tabular}{|c|c|c|c|c|c|}
\hline Mutations & Values & $\begin{array}{c}\text { MoE Absent (n:10167) } \\
\text { \% (n) }\end{array}$ & $\begin{array}{c}\text { MoE Present (n:1041) } \\
\text { \% (n) }\end{array}$ & $\begin{array}{c}\text { Total (n:11208) } \\
\text { \% (n) }\end{array}$ & p \\
\hline \multirow[t]{2}{*}{14408} & Absent & 38.4 (3905) & $27.7(288)$ & 37.4 (4193) & \multirow{2}{*}{$<0.001 *$} \\
\hline & Present & $61.6(6262)$ & $72.3(753)$ & $62.6(7015)$ & \\
\hline \multirow[t]{2}{*}{14805} & Absent & 89.6 (9113) & $94.3(982)$ & 90.1 (10095) & \multirow{2}{*}{$<0.001^{*}$} \\
\hline & Present & $10.4(1054)$ & $5.7(59)$ & $9.9(1113)$ & \\
\hline \multirow[t]{2}{*}{15324} & Absent & 97.0 (9866) & 99.6 (1037) & 97.3 (10903) & \multirow{2}{*}{$<0.001 *$} \\
\hline & Present & $3.0(301)$ & $0.4(4)$ & $2.7(305)$ & \\
\hline \multirow[t]{2}{*}{13730} & Absent & 99.1 (10074) & 99.8 (1039) & $99.2(11113)$ & \multirow{2}{*}{$0.011 *$} \\
\hline & Present & $0.9(93)$ & $0.2(2)$ & $0.8(95)$ & \\
\hline \multirow[t]{2}{*}{14786} & Absent & 99.2 (10089) & 99.7 (1038) & 99.3 (11127) & \multirow{2}{*}{0.085} \\
\hline & Present & $0.8(78)$ & $0.3(3)$ & $0.7(81)$ & \\
\hline \multirow[t]{2}{*}{13536} & Absent & 99.5 (10112) & 99.9 (1040) & 99.5 (11152) & \multirow{2}{*}{0.060} \\
\hline & Present & $0.5(55)$ & $0.1(1)$ & $0.5(56)$ & \\
\hline \multirow[t]{2}{*}{13862} & Absent & $99.6(10128)$ & 99.4 (1035) & 99.6 (11163) & \multirow{2}{*}{0.349} \\
\hline & Present & $0.4(39)$ & $0.6(6)$ & $0.4(45)$ & \\
\hline \multirow[t]{2}{*}{13627} & Absent & $99.6(10130)$ & 99.9 (1040) & 99.7 (11170) & \multirow{2}{*}{0.256} \\
\hline & Present & $0.4(37)$ & $0.1(1)$ & $0.3(38)$ & \\
\hline \multirow[t]{2}{*}{14877} & Absent & 99.6 (10129) & $100.0(1041)$ & 99.7 (11170) & \multirow{2}{*}{-} \\
\hline & Present & $0.4(38)$ & - & $0.3(38)$ & \\
\hline \multirow[t]{2}{*}{15540} & Absent & $99.6(10130)$ & $99.9(1040)$ & 99.7 (11170) & \multirow{2}{*}{0.256} \\
\hline & Present & $0.4(37)$ & $0.1(1)$ & $0.3(38)$ & \\
\hline
\end{tabular}


Table 2 (on next page)

Distribution of MoE across geographical locations.

*p-value $<0.05$ was statistically significant. 
Table 2. Distribution of MoE across geographical locations.

\begin{tabular}{|c|c|c|c|c|}
\hline \multirow{3}{*}{ Locations } & Moe Absent & Moe Present & Total & \multirow{3}{*}{$\mathbf{p}$} \\
\hline & & & & \\
\hline & $\%(n)$ & $\%$ (n) & $\%(n)$ & \\
\hline Africa & $91.5(65)$ & $8.5(6)$ & $100.0(71)$ & \multirow{7}{*}{$<0.001^{*}$} \\
\hline Asia & $92.0(790)$ & $8.0(69)$ & $100.0(859)$ & \\
\hline Europe & $88.6(5111)$ & $11.4(658)$ & $100.0(5769)$ & \\
\hline North America & 94.8 (3194) & $5.2(176)$ & $100.0(3370)$ & \\
\hline Oceania & $88.5(904)$ & $11.5(117)$ & $100.0(1021)$ & \\
\hline South America & 87.3 (103) & $12.7(15)$ & $100.0(118)$ & \\
\hline Total & 90.7 (10167) & $9.3(1041)$ & $100.0(11208)$ & \\
\hline
\end{tabular}

2 $*$ p-value $<0.05$ was statistically significant. 


\section{Table 3(on next page)}

Logistic regression model of MoE and location on single variables. Each location was represented as itself (1) and others (0).

OR, Odds-Ratio; C.I.: confidence interval, *p-value $<0.05$ was statistically significant. 
1 Table 3. Logistic regression model of $\mathrm{MoE}$ and location on single variables. Each location was 2 represented as itself (1) and others (0).

3

4

\begin{tabular}{c|ccl}
\hline Locations & $\mathbf{p}$ & OR & $\mathbf{9 5 \%}$ C.I. \\
\hline Africa & 0.807 & 0.901 & 0.389 to 2.084 \\
Asia & 0.188 & 0.843 & 0.653 to 1.087 \\
Europe & $<0.001 *$ & 1.700 & 1.490 to 1.939 \\
North America & $<0.001^{*}$ & 0.444 & 0.376 to 0.525 \\
Oceania & $0.012^{*}$ & 1.297 & 1.058 to 1.591 \\
South America & 0.200 & 1.428 & 0.828 to 2.465 \\
\hline
\end{tabular}

5

OR, Odds-Ratio; C.I.: confidence interval, *p-value $<0.05$ was statistically significant.

6 


\section{Table 4 (on next page)}

Logistic regression model of MoE on single variables and a final model. (Final Model A) Model of four mutations on final model (Final Model B) Model of four mutations and location on final model

OR, Odds-Ratio; C.I.: confidence interval; Multiple logistic regression final model was executed on all these statistically significant variables, included together in the model, and selected with backward stepwise method; *p-value $<0.05$ was statistically significant. 
1 Table 4. Logistic regression model of MoE on single variables and a final model. (Final Model A) Logistic regression model of four

2 mutations on final model (Final Model B) Logistic regression model of four mutations and location on final model

\begin{tabular}{c|ccl|ccc|ccc}
\hline & \multicolumn{3}{|c|}{ Single Variables } & \multicolumn{3}{c|}{ Final Model A } & \multicolumn{3}{c}{ Final Model B } \\
\hline Mutations & p & OR & $\mathbf{9 5 \%}$ C.I. & p & OR & $\mathbf{9 5 \%}$ C.I. & p & OR & 95\% C.I. \\
\hline n14408 & $<0.001^{*}$ & 1.630 & 1.415 to 1.878 & $<0.001^{*}$ & 1.522 & 1.305 to 1.776 & $0.004^{*}$ & 1.282 & 1.082 to 1.519 \\
n14805 & $<0.001^{*}$ & 0.519 & 0.396 to 0.681 & $0.008^{*}$ & 0.673 & 0.502 to 0.903 & $<0.001^{*}$ & 0.478 & 0.352 to 0.648 \\
n15324 & $<0.001^{*}$ & 0.126 & 0.047 to 0.340 & $<0.001^{*}$ & 0.108 & 0.040 to 0.290 & $<0.001^{*}$ & 0.089 & 0.033 to 0.240 \\
n13730 & $0.028^{*}$ & 0.209 & 0.051 to 0.847 & $0.049^{*}$ & 0.243 & 0.060 to 0.992 & $0.025^{*}$ & 0.201 & 0.049 to 0.820 \\
n14786 & 0.095 & 0.374 & 0.118 to 1.186 & - & - & - & - & - & - \\
n13536 & 0.086 & 0.177 & 0.024 to 1.279 & - & - & - & - & - & - \\
n13862 & 0.352 & 1.505 & 0.636 to 3.564 & - & - & - & - & - & - \\
n13627 & 0.188 & 0.263 & 0.036 to 1.921 & - & - & - & - & - & - \\
n14877 & 0.998 & 0.000 & 0.000 to - & - & - & - & - & - & - \\
n15540 & 0.188 & 2.263 & 0.036 to 1.921 & - & - & - & - & - & - \\
Location & $<0.001 *$ & - & - & & & - & $<0.001^{*}$ & & \\
Africa & 0.901 & 1.057 & $0.442-2.527$ & - & - & - & 0.574 & 1.292 & $0.528-3.160$ \\
South America & 0.092 & 1.667 & $0.920-3.023$ & - & - & - & 0.263 & 1.416 & $0.770-2.605$ \\
Europe & $0.003^{*}$ & 1.474 & $1.138-1.910$ & - & - & - & $0.036^{*}$ & 1.353 & $1.020-1.794$ \\
North America & $0.002^{*}$ & 0.631 & $0.473-0.842$ & - & - & - & $<0.001^{*}$ & 0.537 & $0.398-0.725$ \\
Oceania & $0.014 *$ & 1.482 & $1.084-2.025$ & - & - & - & $0.026^{*}$ & 1.448 & $1.045-2.008$ \\
\hline
\end{tabular}




\section{Figure 1}

Bar graph of top 50 most mutated nucleotides vs. log2-transformed number of samples with non-reference nucleotide at position.

The $x$-axis represents the position of the nucleotide in the reference genome, the $y$-axis represents log 2 of number of isolates with disagreeing nucleotide aligning to the position in sequence plus 1. Unresolved sequence calls during library sequencing or gaps are not included in the number of isolates. Colors of the bars indicate the gene locus or mature peptide region where the nucleotide is in, with the $\mathrm{RdRp}$ mature peptide being considered separately from the remainder of the Orflab region. The 5' untranslated region and the 3'most 100 nucleotides are not included in the graph.

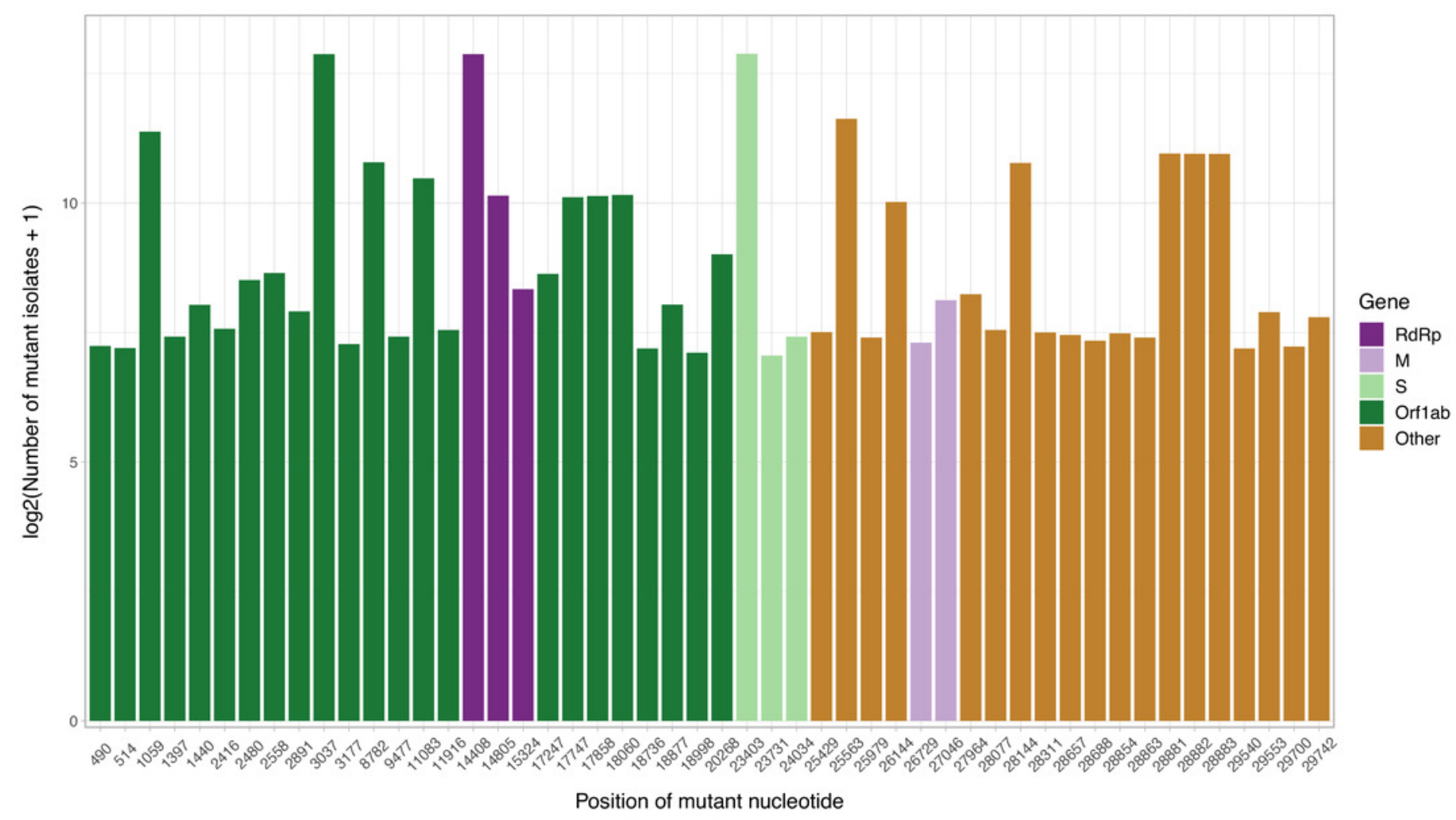


Figure 2

Bar graph of percent of isolates per region containing non-reference coding sequences for the proteins envelope glycoprotein (E), membrane glycoprotein (M), and RdRp protein.

Violet bars represent percent of isolates with mutant envelope glycoprotein, while orange bars represent the same percentage for membrane glycoprotein, and green bars represent the same for RdRp. 


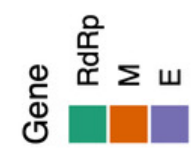
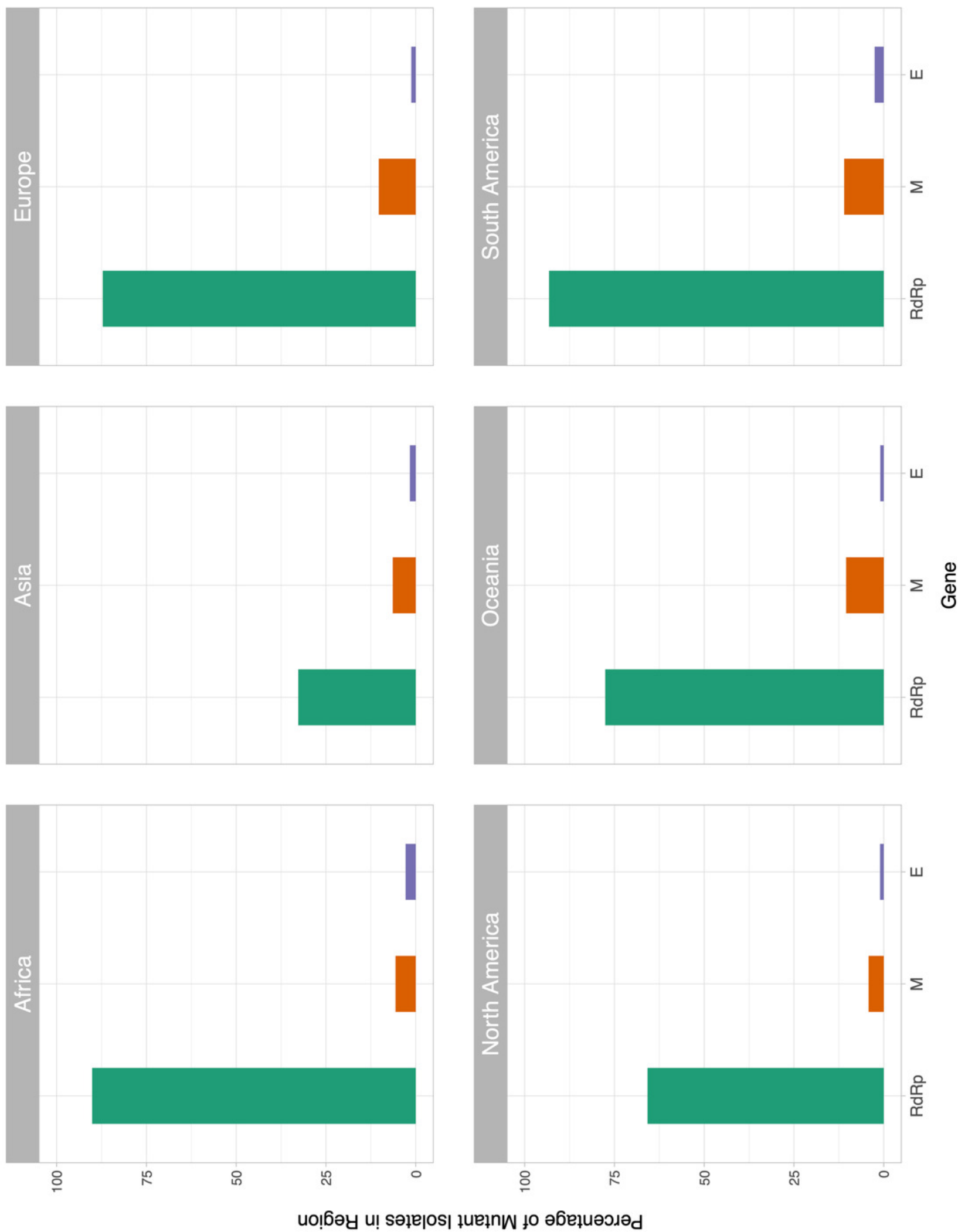